\title{
SEMBLANZAS
}

\section{EN HONOR DE LA JUBILACIÓN DE ROBERT I. BURNS, S. J.}

Robert I. Burns, S.J. al terminar el curso académico de 1990-1991 se despidió oficialmente de su carrera profesional en la Universidad de California de Los A'ngeles; sin embargo, su jubilación no afecta mucho su tarea docente porque con la dedicación que siempre ha mostrado para todo lo que se relaciona con su vida de profesor, continuará enseñando en seminarios especializados durante el año lectivo 1991-1992, y dirigiendo estudios doctorales aun después de 1992. No obstante, espera tener más tiempo para dedicarlo a la gan pasión de su vida, el descubrimiento de documentos sobre la Valencia del siglo XIII.

La gran ventaja de esta jubilación oficial consiste en la oportunidad que nos ofrece para reconocer públicamente la deuda que tenemos todos los historiadores a los esfuerzos del Padre Burns cuya investigación y méritos académicos durante su larga carrera son realmente excepcionales, y, que, en nuestra opinión, difícilmente llegarán a repetirse en el futuro. Su contribución, valiosa y excepcional, no se limita solamente a la publicación del resultado de su investigación diligente en los archivos españoles sino en fomentar el interés de sus compatriotas norteamericanos por la historia del Mediterráneo Occidental. Es imposible evaluar debidamente una carrera tan activa, variada y distinguida, así, a pesar de que no acostumbramos a emplear una sobreabundancia de superlativos, en esta ocasión nos encontramos sin adejtivos suficientemente fuertes para describir adecuadamente la extendida influencia de este historiador; una influencia contínua y provechosa que ha dejado su huella indeleble, tanto en las Universidades de California, donde ha desempeñado su vida docente, como entre los estudiosos norteamericanos y europeos donde sus obras se consideran fundamentalmente para conocer la historia de la Corona de Aragón y, resulta superfluo decirlo, para apreciar el papel de Jaime I en el reino de Valencia. Es imprescindible notar aquí también que este campo de especialización no es el único al que se ha dedicado el Padre Burns, cuya primera tesis doctoral fue escrita sobre los indios norteamericanos. La obra que se publicó como consecuencia de estos estudios también se considera básica para el conocimiento de los primeros habitantes de América del Norte y, de vez en cuando, todavía se le invita al Padre Burns a pronunicar conferencias sobre este tema. 
Su carrera ha sido tan distinguida que somos conscientes de la imposibilidad de dar en pocas líneas una idea de su contribución a la historia del siglo XIII. Esta devoción no habría sido suficiente en sí misma, sin la inteligencia e imaginación con que se ve dotado el Padre Burns. De un documento que al parecer resulta seco y poco interesante, el Padre Burns es capaz de hacer todo un artículo, lleno de detalles divertidos y con una visión muy amplia del particular; para nosostros, ésta es la señal de un genio y no dudamos de que así se le debería considerar. Por ésto, somos muy conscientes de lo inadecuado de nuestras palabras, esperando solamente que contribuyen a hacer resaltar el valor de la labor intensa del ilustre religioso.

Es evidente que este Homenaje tendrá que ser selectivo porque, a diferencia de la mayoría de historiadores, la obra del Padre Burns es extraordinariamente abundante y diversa. Por eso hemos pensado que atendiendo al interés de la mayor parte de los lectores del ANUARIO, será mayor que nos limitemos a hablar de sus principales trabajos sobre la Corona de Aragón, y especialmente sobre el reino de Valencia. Al tomar esta decisión vamos a dejar sin comentar una parte importante de su producción, tanto en el campo de la historia como en la vida universitaria y académica y, con el fin de indicar la extensión de la obra del Padre Burns, intentaremos resumir en pocos párrafos algunas de estas muchas contribuciones. Al final de nuestro comentario daremos una bibliografía selecta de los libros y artículos del Padre Burns sobre la historia de la Corona de Aragón, indicando también unos libros y artículos de próxima publicación.

Reparamos un momento en la persona del Padre Burns, quien para muchos lectores de este ANUARIO es el investigador sentado en el Archivo de la Corona de Aragón, absorbido totalmente en un registro de Jaime I, haciendo uso de una lupa potente para descifrar lo indescifrable, el bloc de papel en la mesa, la mano preparada para escribir sin perder tiempo. Para los que no le conocen, puede parecer algo enigmático, reservado y hasta tímido, aunque siempre afable, cortés, correcto y dotado de una gran humanidad que esconde bajo su aspecto serio de religioso jesuíta. Además, tiene un marcado sentido del humor que le permite disfrutar de muchas situaciones cómicas y hasta le ayuda a descansar de sus horas de trabajo. Su energía infatigable, el día interminable de trabajo a que se somete, no le impiden de interesarse por los problemas de los demás, ni por los asuntos del mundo que le rodea. Con su insaciable afán de leer, consigue tener tiempo para dedicarse a la lectura de placer -por ejemplo, nunca acaba el día sin leer algo relacionado con su trabajo- y, cuando puede, se deleita viendo películas de cine o televisión y, en Barcelona, dando un paseo hacia la Diagonal por su calle predilecta, el Paseo de Gracia. Al describir las aventuras de un investigador en los diversos archivos europeos, le gusta exagerar y hacer reir a sus oyentes y, como narrador, sobresale por su capacidad de recrear una escena o un acontecimiento y convertirlo en una anécdota 
divertidísima, una técnica con que siempre logra mantener la atención del público.

Antes de hablar más detalladamente de las publicaciones del Padre Burns, creemos que es importante hacer resaltar otra contribución suya a la historia medieval de la Corona de Aragón, es decir, la organización de la Biblioteca del "Institute of Medieval Mediterranean Spain» que él mismo fundó en Los Angeles y que continua dirigiendo. Esta Biblioteca especializada es única en la América del Norte por su valiosa colección de libros dedicados a temas de la España medieval. La diligencia y entusiasmo con los que el Padre Burns se ha dedicado a la compra de libros, recorriendo las muchas librerías españolas y asegurándose de que no se le escapara ningún libro de posible utilidad para estudiantes del tema, en si misma merece un homenaje y consta como una gran contribución a la expansión de los estudios de la Corona de Aragón.

A continuación, dividiremos nuestro comentario sobre la carrera del Padre Burns en secciones dedicadas a temas especializadas. Cada una se refiere a una parte de su Curriculum que por ser muy largo resulta imposible reproducir en su totalidad aquí. Para más información sobre sus actividades, remitimos a nuestros lectores a Who's Who in America, Répertoire Internatinale des Médiévistes, Reference Encyclopedia of the American Indian, Repertorio de Medievalismo Histpánico, American Catholic Who's Who y Contemporary Authors, todos los cuales han dedicado páginas a la vida y obra del Padre Burns.

\section{Formación profesional}

La formación del Padre Burns en este campo es mucho más completa de lo normal: aparte del estudio de la Teología, necesaria para su profesión como jesuíta, realizó dos doctorados en Historia, uno en la Universidad de Friburg en Suiza, sobre los Indios de la América del Norte y otro en la Universidad de Johns Hopkins, en los Estados Unidos, sobre Jaime I y la Iglesia valenciana de la Edad Media. No hace falta decir que en ambas Universidades se doctoró con distinción y que los conocimientos adquiridos con el estudio de asignaturas muy diferentes como son Filosofía, Teología e Historia, contribuyeron a su singular éxito. Para completar su preparación en el campo de la Historia Medieval y Moderna, pasó un año en Oxford y otro en Nueva York (Columbia), perfeccionando sus conocimientos de Teología durante otro año en Florencia (Italia). Estos estudios preparativos duraron más de quince años, es decir desde 1945 a 1961, una larga época durante la cual también enseñaba en la Universidad de San Francisco y en la Universidad Gregoriana de Roma. No es extraño, entonces, que su carrera posterior siguiera un camino vertiginoso de publicaciones, honores y labor intensa. 


\section{Años docentes}

No encontramos las palabras adecuadas para elogiar, con el entusiasmo que sentimos, la dedicación del Padre Burns a la enseñanza y a las responsabilidades que la acompañan. Durante los casi treinta años que pasó en su ciudad nativa, San Francisco, se le exigieron muchas horas semanales, la gran mayoría de ellas en la preparación de estudiantes para la licenciatura y no en la enseñanza de temas de especial interés para él. Como la Universidad de San Francisco no tenía ninguna sección de estudios doctorales en Historia Medieval, los que querían cursar el doctorado con el Padre Burns no podían hacerlo y sólo cuando se trasladó a la Universidad de California en Los Angeles le fue posible dedicarse a la preparación de candidatos doctorales. Fue en el año 1967, después de haberse negado varias veces a dejar su querido San Francisco, que el Padre Burns, finalmente, se decidió a aceptar la Cátedra de Historia Medieval en la Universidad de California en Los Angeles. A partir de esta fecha, siempre ha tenido estudiantes dedicados a temas de Historia de la Corona de Aragón y, cada año, aumenta el número de los que se quieren beneficiar de su experiencia, ayuda y dirección. En el momento de su jubilación, tiene unos veinte estudiantes que siguen estudios sobre aspectos de la Edad Media bajo su dirección, y que continuarán con él hasta que completen su doctorado.

A pesar de haber dejado muy clara su decisión de no querer cambiar de Universidad, nunca faltaban los que intentaban hacerle aceptar Cátedras muy honoríficas en sus Universidades. El padre Burns agradeció a todos la atención e insistió en que no pensaba trasladarse de Los A'ngeles, donde tenía todas las facilidades para la preparación de candidatos doctorales y donde también disponía de un buen local para su Instituto.

\section{Actividades profesionales}

En 1962, un año después de doctorarse en Friburg, y tres depués de recibir la Summa en Historia Medieval de Johns Hopkins, llegó a ocupar la posición de vice-presidente de la Sociedad Americana de Historia de la Iglesia («Society for American Church History») sucursal occidental, y durante los años siguientes desempeñó un papel activo en las principales sociedades profesionales de su disciplina en América del Norte. En 1975, fue Presidente de la Asociación Americana de Historiadores Católicos («American Catholic Historical Association») cuya organización tiene unos 2.000 miembros y cuyas actividades incluyen la publicación de la revista "The Catholic Historical Review». Tres años más tarde le hicieron Vicepresidente de la Asociación Americana de Historia («American Historical Association») -sucursal de la costa pacífica), ocupando el cargo de Presidente en el año 1979. Durante estos años también 
fundó otra organización importante para la Historia Medieval de España, la Academia Americana de Investigadores de la España Medieval («American Academy of Research Historians of Medieval Spain»), siendo Presidente desde 1976 hasta 1978, en cuyo año le eligieron miembro de la Junta de Govern de la «Societat Nord Americana de Estudis Catalans» («North American Catalan Society") para representar a los historiadores, y podríamos continuar citando su representación en todas las organizaciones importantes que se dedican a la enseñanza y a la difusión de la Historia, pero los interesados pueden consultar su Curriculum en alguna de las obras mencionadas arriba.

\section{Publicaciones}

Es aquí donde el Padre Burns destaca como historiador sumamente distinguido y, casi podríamos decir, como pionero en el descubrimiento de las riquezas contenidas en el Archivo de la Corona de Aragón para comprender la conquista de Valencia por Jaime I y para apreciar el desarrollo del reino de Valencia en el siglo XIII. No se ha limitado a este tema pero los historiadores del futuro siempre le recordarán como investigador del reino de Valencia. El hecho de que se esté traduciendo toda su obra al catalán y que la Universidad de Valencia le haya nombrado Doctor "honoris causa" es prueba palpable de su posición eminente en el campo de la Historia Medieval de Valencia. No podemos mencionar aquí todas sus publicaciones porque necesitariamos más espacio del destinado a este artículo y, además, las más importantes son bien conocidas por todos los historiadores dedicados a temas de Historia Medieval de la Corona de Aragón. No obstante, nos parece útil indicar la enorme producción del distinguido investigador y señalar algunas de sus obras fundamentales: un total de 50 libros entre los cuales se incluyen traducciones al castellano o al catalán de libros publicados originalmente en inglés, todos ellos revisados y puestos al día, más de 100 artículos, capítulos de libros y contribuciones a enciclopedias, y otros reportajes, reseñas, comunicaciones a Congresos etc., evidencia de una erudición alarmante y hasta inhibitoria para los que siguen sus pasos por los caminos del Archivo de la Corona de Aragón.

La mayoría de sus libros tienen como tema principal Jaime I y el reino de Valencia; nos limitamos a mencionar algunos de los principales Crusader Kingdom of Valencia, Islam under the Crusaders, Medieval Colonialism, Moors and Crusaders in Medieval Spain, Jaume I, Muslims, Cbristians and Jews in Crusader Valencia y Society and Documentation in Crusader Valencia. No obstante, su primer libro que queda hoy como estudio fundamental para los que estudian los indios de la América del Norte, Jesuits and Indian Wars, salió en edición revisada en 1985, veinte años después de haber embarcado su autor sobre otro tema de investigación, el del reino de Valencia durante el siglo XIII, 
demostrando la gran capacidad del Padre Burns para cualquier tarea intelectual y para dedicarse a temas y siglos muy diferentes sin ninguna dificultad.

El Diplomatarium que lleva años preparando y que consiste en una colección de documentos sobre el reino de Valencia en el siglo XIII (Documentation in Crusader Valencia es el primer tomo) es fruto de las muchas horas que ha pasado en el Archivo de la Corona de Aragón, estudiando con suma atención cada letra, punto y lacuna de las cartas contenidas en los registros de la cancillería de Jaime I. El primer volumen explica la naturaleza de la cancilleria, la técnica de los escribanos y otros muchos detalles sobre la producción del papel que hizo posible tan minuciosa conservación de documentos söbre los acontecimientos importantes para el reino y para el mismo Jaime I.

El segundo volumen, la noticia de cuya publicación por Princeton nos llega al escribir estas páginas, consiste en las transcripciones de unos 500 documentos hechas con mucho cuidado y repasadas contínuamente hasta que él mismo se queda satisfecho de la corrección del producto final, labor que le lleva a angustiarse a veces sobre una letra o el significado de la carta, porque su máxima aspiración es no dejar nada en blanco y reproducir fielmente las palabras escritas en el registro de cancillería. La dificultad de conseguirlo la conocen todos los investigadores que pasan horas en los archivos de la Edad Media, y muchos se asustan ante la gran capacidad del Padre Burns a descifrar letras borrosas, otras a medio desaparecer, muchas abreviaturas difíciles o, por ser muy pequeñas, nada fáciles de leer. No obstante, el Padre Burns llega a dar sentido a lo que parece imposible de descifrar y el gran cuidado que tiene en reproducir fielmente el documento que transcribe es la característica de un investigador poco usual, paciente y con singular dedicación, cualidades que aporta el Padre Burns a todo lo que hace. Sus visitas anuales a Barcelona se consumen en el Archivo de la Corona de Aragón donde realiza la verificación de sus documentos para el Diplomatarium y donde continua buscando novedades para publicar sobre el siglo XIII. Es de notar que siempre tiene tiempo para ayudar a los estudiantes o a los neófitos que encuentra en el Archivo y para establecer contactos con otros Norteamericanos que consultan los documentos. Hoy día no nos extraña ver a historiadores de conocida reputación aprovecharse de la experiencia del Padre Burns y todos los que se dedican al siglo XIII quieren consultarle sobre su investigación porque saben que no hay nadie que conozca los documentos del Archivo de la Corona de Aragón como él.

\section{Libros en preparación}

No contento con la elaboración de un Diplomatarium en muchos volúme- 
nes, el Padre Burns tiene preparado otro libro fundamental para la Historia Medieval, una historia de la Orden de Penitencia de Jesucristo, cuyos miembros son conocidos como los «Frailes del Sac», y cuyas actividades hasta ahora se han visto envueltas en el misterio. El Padre Burns ha examinado colecciones de documentos en muchos Archivos europeos y españoles y ha podido sacar conclusiones sobre la vida de estos frailes desaparecidos poco después de su fundación en el siglo XIII, pero cuya huella se encuentra en la Orden de Frailes Menores. Suprimidos en 1274, continuaron ejerciendo mínimamente su misión hasta la muerte del último de ellos durante la primera mitad del siglo XIV. El artículo que publicó el Padre Burns en el Dizionario degli Istituti di Perfezione es el único estudio sobre estos frailes, cómo venían por los caminos de la Europa medieval vestidos con hábitos que recordaban sacos, siempre pobres y mendigando para comer y para sostenerse. Esperamos con entusiasmo su libro definitivo sobre el asunto.

\section{Conferencias, comunicaciones y reseñas}

A pesar de las muchas horas dedicadas a la investigación y otras muchas a leer obras de toda clase - no se ve nunca al Padre Burns sin un libro en las manos- ha sido buscado y solicitado contínuamente para dar conferencias, asistir a Congresos y Simposios y hacer reseñas de libros publicados sobre Historia Medieval. A veces, para cumplir con estas peticiones se ha visto obligado a atravesar los Estados Unidos o a cruzar el Atlántico, viajes que suponen muchas horas de vuelo y la consiguiente pérdida de tiempo. Cada año, como muestra su Curriculum, ha dedicado un sin fin de horas a hablar sobre diversos temas de su primordial interés. Casi todas estas conferencias son borradores de un capítulo alguno de sus libros o de un artículo que después publica en revistas como «Speculum», "Catholic Historical Review», «English Historical Review», "Anuario de Estudios Medievales», "Sharq-al-Andalus», etc. etc., sin contar con su leal apoyo a cada Congreso de Historia de la Corona de Aragón y a cada reunión de la "American Historical Association».

\section{Honores y premios}

La investigación del Padre Burns le ha ganado varios honores y premios, tanto en los Estados Unidos como en España. Entre ellos se destacan los doctorados «honoris causa» de las siguientes Universidades: Valencia (1985), Marquette (1977), Loyola, Chicago (1976), Boston College (1982), San Francisco (1983), Fordham, New York (1984), Gonzaga (1968), Georgetown (1982), una serie de honores tan extraordinaria que no deja ninguna duda de la 
importancia que se da a la contribución del erudito Padre Burns como historiador. No podemos mencionar todos los premios que ha recibido durante su distinguida carrera pero los más significativos para su investigación en la Corona de Aragón son los más recientes, es decir el Premi de la Crítica «Serra d'Or» (1982), «Premi Catalònia» del Institut d'Estudis Catalans (1982), «Premi Internacional Llull» (1988) i la "Creu de Sant Jordi» (1989». En los Estados Unidos, le han otorgado premios de la «Catholic Historical Association", la "American Historical Association", la "Medieval Academy of America" y le han reconocido su labor en Congresos científicos, revistas especializadas y en muchas otras ocasiones, evidenciando, una vez más, la gran envergadura que ha tenido internacionalmente la obra de este gran historiador. Sin embargo, estos honores y premios no se limitan a su labor investigadora sino que se extienden al campo de la enseñanza, donde también ha obtenido una serie impresionante de galardones.

\title{
Becas concedidas
}

Desde el inicio de su carrera ha recibido becas de todas las importantes fundaciones americanas: he aquí algunas de ellas: Guggenheim, Ford, National Endowment for the Humanities, Del Amo, American Council of Learned Societies, Princeton Institute for Advanced Study, Rockefeller; y de España: Consejo Superior de Investigaciones Científicas, Promoció de Cultura Valenciana y del tratado cultural entre Estados Unidos y España, algunas de las cuales fueron concedidas más de una vez, prueba de su reputación distinguida y notable.

Finalmente, creemos que la disciplina de la vida religiosa y la gran capacidad intelectual del Padre Burns, junto con el interés que siempre ha tenido para la historia de la civilización, tanto de su propio país como de la Edad Media en el Mediterráneo, le han convertido en uno de los más distinguidos historiadores de todas las épocas y, a la vez, en un colega excepcional: le deseamos muchos años más de productividad en la preparación y publicación de libros y esperamos que continue disfrutando de la buena salud necesaria para dedicarse plenamente a los múltiples proyectos que sabemos que tiene entre manos.

Septiembre, 1991

\author{
JILL R. WEBSTER, \\ St. Michael's College, University of Toronto
}




\section{BIBLIOGRAFÍA SELECTA}

Libros:

The Crusader Kingdom of Valencia: Reconstruction on a Thirteenth-Century Frontier, Cambridge, Harvard University Press, 1967, 2 vols. XVIII + 561 pp. Versión castellana revisada y ampliada $E l$ reino de Valencia en el siglo XIII (iglesia $y$ sociedad), Valencia, Del Cénia al Segura (Promoció de Cultura Valenciana), 1982, 2 vols. XII, 801 pp. Versión catalana revisada El regne croat a València, València, Tres i Quatre, en prensa.

Islam under the Crusaders: Colonial Survival in the Thirteenth-Century Kingdom of Valencia, Princeton, Princeton University Press, 1974, XXXII + 475 pp. Versión catalana revisada L'Islam sota els croats, 2 vols., València, Tres i Quatre, 1990

Medieval Colonialism: Postcrusade Exploitation of Islamic Valencia, Princeton, Princeton University Press, 1976, XXXV + 394 pp. Versión catalana revisada

Colonialisme medieval: Explotació postcroada de la València islàmica, Biblioteca d'Estudis i Investigacions, núm. 9, València, Tres i Quatre, 1987, XV + 485 pp.

Moors and Crusaders in Mediterranean Spain Collected Studies, London, Variorum, 1978,318 pp.

Jaume I i els valencians del segle XIII, Valencia, Tres i Quatre (Biblioteca d'Estudis i Investigacions), $1981, \mathrm{XI}+348 \mathrm{pp}$.

Muslims, Cbristians and Jews in the Crusader Kingdom of Valencia: Societies in Symbiosis, Cambridge University Press, 1984, 363 pp. Versión catalana Moros, cristians $i$ jueus en el regne croat de València: Societats en simbiosi, Valencia, Tres $i$ Quatre, Biblioteca d'Estudis i Investigacions, núm. 13 (1987), 489 pp.

Society and Documentation in Crusader Valencia, Princeton, Princeton University Press, 1985, XI + 275 pp. Versión catalana Societat $i$ documentació en el regne croat de València, València, Tres i Quatre, Biblioteca d'Estudis i investigacions, 14 (1984).

The Foundation of Crusader Valencia: Revolt and Recovery, Diplomatarium of the Crusader Kingdom of Valencia, vol. 2 (Princeton University Press, 1990), 474 pp. 
Libros en preparación muy adelantada:

The Friars of the Sack in the Realms of Aragon.

Jaume the Conqueror: Years of Triumph, Years of War 1264-1270, título provisional, es el tomo 3 del Diplomatarium.

Libros editados:

Alfonso the Learned, Emperor of Culture, 1284-1984, New York, Fordham University Press, 1985.

The Worlds of Alfonso the Learned and James the Conqueror: Intellect and Force in the Middle Ages, Princeton, Princeton University Press, XXV + 233 pp. Versión castellana Los mundos de Alfonso el Sabio y Jaime el Conquistador: Razón y fuerza en la Edad Media, Edicions Alfons el Magnànim, Institució Valenciana d'Estudis i Investigació, 1990.

Articulos y capitulos en libros:

The Catalan Company and the European Powers, 1305-1311, "Speculum», XXIX (1954), pp. 751-771.

Un monasterio-hospital del siglo XIII: San Vicente de Valencia, "Anuario de Estudios Medievales», IV (1967), pp. 75-108.

Irrigation Taxes in Early Valencia: The Problem of the Alfarda, "Speculum», XLIV (1969), pp. 560-567.

Baths and Caravanserais in Crusader Valencia, "Speculum», XIVI (1971), pp. 443-458.

Christian-Islamic Confrontation in the West: The Thirteenth Century Dreams of Conversion, "American Historical Review», LXX (1971), pp. 1386-1434.

How to End a Crusade: Techniques for Making Peace, "Military Affairs», XXXV (1971), pp. 142-148.

Renegades, Adventurers and Sharp Businessmen: The Thirteenth-Century Spaniard in the Cause of Islam, "Catholic Historical Review», LVII (1972), pp. 341-366.

The Medieval Crossbow as Surgical Instrument: An Illustrate Case History, "Bulletin of New York Academy of Medicine», XLVIII (1972), pp. 982-989.

Le royaume chrétien de Valence et ses vassaux musulmans (1240-1280), "Annales: Économies, Sociétés, Civilisations», XXVIII (1973), pp. 199-225.

Islam [as an Established Religion] in the Kingdom of Valencia, en "Studies in Medievalia and Americana: Essays in Honor of William Lyle Davis S.J., ed. G.R. Steckler and L.D. Davis, Spokane, Gonzaga University Press, 1973, pp. 1-34. 
Immigrants from Islam: The Crusaders'Use of Muslims as Settlers in Thirteenth-Century Spain, "American' Historical Review», LXXIV (1975), pp. 22-42.

Spanish Islam in Transition: Acculturative Survival and its Price in the Christian Kingdom of Valencia, 1240-1280, en "Islam and Cultural Change in the Middle Ages, ed. Speros Vryonis, Jr., Wiesbaden, Germany, Otto Harrassowitz [and UCLA Near Eastern Center], (1975), pp. 87-105.

The Spiritual Life of James the Conqueror, King of Arago-Catalonia, 1208-1276: Portrait and Self-Portrait, Catholic Historical Review», LXII (1976), pp. 1-35.

The Muslim in the Christian Feudal Order: The Kingdom of Valencia, 1240-1280, "Studies in Medieval Culture», V (1976), pp. 105-126-

The Realms of Aragon: New Directions in Medieval History, "Midwest Quarterly", XVIII (1977), pp. 225-239.

The Language Barrier: The Problem of Bilingualism and Muslim-Christian Interchange in the Medieval Kingdom of Valencia", in Contributions to Mediterranean Studies, ed. Mario Vassallo, Malta, Royal University of Malta, 1977, pp. 116-136.

Mudejar History Today: New Directions, "Viator», VIII (1978), pp. 127-143.

Muslim-Christian Conflict and Contact in Medieval Spain: Context and Metbodology, "Studies in Honor of Robert McNally", ed. G. R. Dimler (commemorative issue of "Thougth», LIV [Fall 1979], as volume), pp. 238-252.

La muralla de la llengua: el problema de bilingüisme i de la interacció entre musulmans $i$ cristians al regne medieval de València, «L'Espill», 1/2 (Valencia, 1979), pp. 15-35.

Piracy as an Islamic-Christian Interface in the Thirteenth Century, "Viator», XI (1980), pp. 165-178.

Societies in Symbiosis: The Mudejar-Crusader Experiment in Thirteenth-Century Mediterranean Spain, "International History Review», II (University of Toronto, 1980), pp. 349-385.

Canon Law and the Reconquista: Convergence and Symbiosis in the Kingdom of Valencia under Jaume the Conqueror (1213-1276), "Proceedings of the Fifth International Congress of Medieval Canon Law», ed. Stephan KUTTNER and Kenneth PENNINGTON, Rome, Biblioteca Vaticana, 1980, pp. 387-424.

Mudejar Life and Work: Colonial Tax Structure in the 13th-Century Kingdom of Valencia, "I Congreso de Historia del País Valenciano» (4 vols., Universidad de Valencia, 1973-1980), II (1980), pp. 435-452.

Los limites interiores de la Valencia de la Reconquista: un género de tipología documental, «Medievalia», I (Universidad de Barcelona, 1980), pp. 9-34. 
Los mudéjares de Valencia: temas y metodología (con apéndice de 52 documentos inéditos), "I Simposio Internacional de Mudejarismo» (Madrid, C.S.I.C., 1981), pp. 453-497.

The Paper Revolution in Europe: Crusader Valencia's Paper Industry, a Technological and Bebavioral Breakthrough, «Pacific Historical Review», L (1981), pp. 1-30.

Socioeconomic structure and Continuity: Medieval Spanish Islam in the Tax Records of Crusader Valencia, "Studies in Economic and Social History" [Princeton University Studies in the Near East] Princeton, 1981 [pp. 838], pp. 251-281.

A Medieval Earthquake: Jaume I, al-Azraq, and the Early History of Onteniente, in C. E. Dufourcq et alii eds., Jaime I y su época, 3 vols. [ «X Congreso de Historia de la Corona de Aragón»], (Zaragoza, C.S.I.C., 1979-1982), II (1982), pp. 209-244.

Jaume $I$ and the Jews of the Kingdom of Valencia, "Jaime I y su época supra», II, pp. 245-322.

The Spiritual Life of Jaume the Conqueror: Portrait and Self-Portrait, "Jaime I y su época supra», II, pp. 323-357, versión revisada.

Relic Vendors, Barefoot Friars and Spanish Muslims: Reflections on Medieval Economic and Religious History, "Comparative Studies in Society and History" [Cambridge University Press], XXIV (1982), pp. 441-442.

Rehearsal for the Sicilian War: Pere el Gran and the Mudejar Countercrusade in the Kingdom of Valencia, 1276-1278, "La società mediterranea all'epoca del Vespro», Francesco Giunta and Petro Corrao eds., 3 vols; «XI Congreso di Storia della Corona d'Aragona», Palermo, Accademia di Scienze, Lettere, e Arti, 19831984, II, pp. 259-287.

[en colaboración con Paul CHEVEDDEN], El tractat de rendició d'Al-Azraq amb Jaume I $i$ l'infant Alfons en 1245: el text àrab i el context valencià, "Homenatge al Professor Manuel Sanchís Guarner", Valencia, Tres i Quatre, 1983, y como artículo en «L'Espill»XVII-XVIII (Valencia 1983), pp. 231-257-

Los mudéjares de la Valencia de las cruzadas: un capitulo olvidado de la bistoria islámica, «Sharq al-Andalus», I (Universidad de Alicante, 1984), pp. 15-34.

Anti-Semitism and Anti-Judaism in Christian History: A Revisionist Thesis, "Catholic Historical Review», LXX (1984), pp. 90-93.

Les Mudéjars du royaume de Valence: une majorité traitée en minorité dans un royaume $d u$ Moyen-Âge, "Minorités et marginaux en France méridionale et dans la peninsule ibérique (VIIe-VIIIe siècles)», Ed. P. TUCOO-CHALA and M. LAMBERTGORGES, Paris, Centre National de la Recherche Scientifique, 1986, pp. 95-118.

The Muslims of Sicily and Valencia, «Proceedings of the American Historical Association», 1985, Ann Arbor, University Microfilms, 1986. 
La conquesta de Valencia: la dimensió mariana, en "Món i misteri de la Festa d'Elx», 2 vols., (València, Generalitat, 1986, pp. 387, pp. 69-76.

The King's Pardon: A Genre in Documentary Typology (Montpellier, Perpignan Barcelona, 1274), "Medievalia», VII (1987), pp. 57-71, y en «Estudios dedicados al profesor Frederic Udina i Martorell», 2 vols., Universidad Autónoma de Barcelona, 1987-1988.

Jews on the Medieval Urban Frontier [Catalonia], "The Sephardim: A Cultural Journey from Spain to the Pacific Coast», ed. Rabbi Joshua STAMPFER, Portland, Or.; Institute for Judaic Studies, 1987, cap. 1 (pp. 22-35).

Principe almobade y converso mudéjar: nueva documentación sobre Abu Zayd, "Sharq al-Andalus», IV (1987), pp. 109-122.

La guerra de al-Azraq de 1249, "Sharq al-Andalus», IV (1987), pp. 253-256.

The Cantigas de Santa Maria as a Research Opportunity in History, "Bulletin of the Cantigueiros de Santa Maria», I (1987), pp. 17-22.

The Mudejars of Medieval Valencia: A Unique Community of Islam, en "Islam: Continuity and Change», ed. Thomas MICHEL, Rome, Centre d'Études pour le Monde Arabe Moderne, 1987, pp. 23-33 y reimpreso en «Salaam», New Delhi, Islamic Studies Association, VIII (1987), pp. 189-198.

The Crusade against al-Azraq: A Thirteenth-Century Mudejar Revolt in International Perspective, "American Historical Review», XCIII (1988), pp. 80-106.

A Lost Crusade: Unpublished Bulls of Innocent IV on al-Azrq's Revolt in ThirteenthCentury Spain, "Catholic Historical Review», LXXIV (1988), pp. 440-449.

The Missionary Syndrome: Crusader and Pacific Northwest Religious Expansionism, "Comparative Studies in Society and History», XXX (Cambridge University, 1988), pp. 271-285.

La manumisión de un musulmán un documento doble de Valencia en 1300, "Sharq al-Andalus», V (1988), pp. 141-145.

The Friars of the Sack in Puigcerdà: A Lost Chapter of 13th-Century Religious History, «Anuario de Estudios Medievales», XVIII (1988), («Homenaje a la memoria del Profesor Emilio Sáez», II), pp. 217-227.

L'Església com a institució fronterera; El rei Jaume I i València: perfil d'un Conqueridor; Els mudéjars del regne de València de la generació posterior a la croada, en "Història del País Valencià», ed. Ernest BelenGuer, 5 vols. (Barcelona, Edicions 62, 1989-1990), II, pp. 125-137; 43-56; 139-167. 
The Loss of Provence: King James's Raid to Kidnap its Heiress (1215) Documenting a Legend, "XIIe Congrès d'Histoire de la Couronne d'Aragon", 3 vols. (Memoires de la Société Archéologique de Montpellier, 15-17), Montpellier: 1987-1989, III, pp. 195-231.

Iglesia y sociedad de Valencia, durante la generación de la conquista, Antecedentes y consecuencias de la conquista de Valencia: En torno al 750 aniversario, 2 vols., Valencia, Consell de Cultura, 1989, II, pp. 97-114.

Regalo para una madre: una muchacha esclava musulmana del nieto de Abu Zayd, el señor de Borriol (1301), "Sharq al-Andalus», VI (1989), pp. 115-117.

The Significance of the Frontier in the Middle Ages, en "Medieval Frontier Societies», ed. Robert BARTLETT and Angus MACKAY, Oxford, Oxford University Press, 1989, pp. 307-330.

Social and Legal History in the Pardon Charters of Jaume the Conqueror (1270-1274), en "Mediterraneo medievale: Scritti in onore di Francesco Giunta", Biblioteca di storia e cultura meridionale, Studi e testi, 2: 3 vols., Soveria Mannelli, Centro di Studi Tardoantichi e Medievali di Altomonte, 1985, I, pp. 213-241.

[En colaboración con Paul CHEVEDDEN], Al-Azraq's Treaty with Jaume I and Prince Alfonso in 1245: Arabic Text and Valencian Context, "Der Islam», LXVI (University of Hamburg, 1989), pp. 1-37.

Muslims in the Thriteenth-Century Realms of Aragon: Interactions and Reaction, en «Muslims under Latin Rule, 1100-1300», ed. James M. POWELL, Princeton University Press, 1990, cap. 2.

Warrior Neighbours: Alfonso el Sabio and Crusader Valencia, an Archival Case Study in International Relations, "Viator», XXI (1990), pp. 147-202.

Articulos en prensa:

A Narrow Window: The Mudejars of the Kingdom of Valencia, 1263-1270, «Islamic Studies», XXIX.

Crime in Medieval Valencia: The Pardon Charters of King Jaume the Conqueror en "Homenatge a Joan Fuster», Universidad de Valencia.

The Della Volta: A Genoese Family in the Service of the Crown of Aragon, 114C-1340, "XIV Congresso di Storia della Corona d'Aragona», Cagliari, 1989.

El naixement d'un poble: el Regne de València, en "Un nou home, un nou món», Generalitat de Valencia, cap. 1 (también aparece en castellano). 
The Daughter of Abu Zayd, Last Almobad Ruler of Islamic Valencia: Her Family and Testament, "Sharq al-Andalus», VII.

\section{RÉSUMÉ}

La mise à la retraite de Robert I. Burns de l'Université de Californie à Los Angeles a été l'occasion de reconnaître son extraordinaire contribution a l'étude de l'histoire médiévale. Ses nombreuses études sur Jaume Ier et sur le treizième siècle valencien, ses nombreux volumes Diplomatarium sur des documents des Archives de la Couronne d'Aragon, la création, en Californie, d'une bibliothèque sur la Méditerranée médiévale espagnole et la préparation des recherches de nombreux étudiants sont l'extraordinaire héritage qu'il nous a légué pendant sa carrière. Joints aux nombreux prix et honneurs qu'il a reçu, sa participation volontaire et importante à des symposiums académiques et le fait qu'il ait porté Valence au premier plan des études historiques, lui garantissent une place permanente parmi les savants distingués du Moyen Age espagnol.

\section{SUMMARY}

The retirement of Robert I. Burns from the University of California at Los Angeles provides an opportunity for recognition of the extraordinary contributions he has made to medieval history. His numerous studies of James I and thirteenth century Valencia, his many volume Diplomatarium of documents from the Crown of Aragon Archives, the creation of a specialized library on medieval Mediterranean Spain in California and the preparation of many research students are the outstanding legacy of his professional career. Together with the many honours and prizes he has received, his willing and significant participation in academic symposia and the fact that he has brought Valencia into the forefront of historical studies can only guarantee him a permanent place among the distinguished scholars of medieval Spain. 